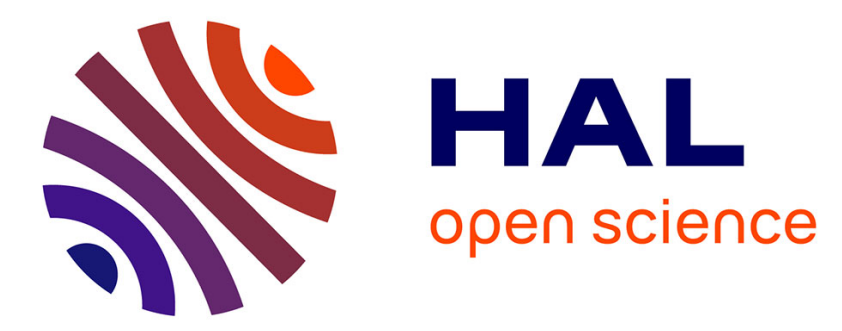

\title{
Propriétés magnétiques du nickel pur à proximité du point de Curie
}

\author{
L. Néel
}

\section{To cite this version:}

L. Néel. Propriétés magnétiques du nickel pur à proximité du point de Curie. J. Phys. Radium, 1935, 6 (1), pp.27-34. 10.1051/jphysrad:019350060102700 . jpa-00233292

\section{HAL Id: jpa-00233292 https://hal.science/jpa-00233292}

Submitted on 1 Jan 1935

HAL is a multi-disciplinary open access archive for the deposit and dissemination of scientific research documents, whether they are published or not. The documents may come from teaching and research institutions in France or abroad, or from public or private research centers.
L'archive ouverte pluridisciplinaire HAL, est destinée au dépôt et à la diffusion de documents scientifiques de niveau recherche, publiés ou non, émanant des établissements d'enseignement et de recherche français ou étrangers, des laboratoires publics ou privés. 


\title{
PROPRIÉTÉS MAGNÉTIQUES DU NIGKEL PUR A PROXIMITÉ DU POINT DE GURIE
}

\author{
Par M. L. NÉEL.
}

Faculté des Sciences de Strasbourg.

\begin{abstract}
Sommaire. -- On décrit un appareil précis de mesure des susceptibilités magnétiques, dans des champs de 50 à 1000 gauss, avec dispositif permettant de repérer les températures à mieux que $0^{\circ} 005$ près. Le nickel a été ainsi étudié à partir du point de Curie $\left(33^{\circ}\right)$ jusqu'à $368^{\circ}$. Dans cet intervalle, on a précisé les variations de la susceptibilité avec la température et le champ magnétique.
\end{abstract}

1. But des expériences. - Les métaux ferromagnéliques n'obéissent à la loi de Curie-Weiss qu'à des températures supérieures de $100^{\circ}$, au moins, au point de Curie. Près du point de Curie, la courbe $\frac{1}{\chi}=f(T)$ est convexe vers l'axe des températures (fig. 1 ). D'ailleurs, à quelques dizaines de degrés au-dessus du point de Curie, la courbure devient très faible et des mesures effectuées dans un petit intervalle de température font croire à l'existence d'une droite. Tout près du point de Curie, au contraire, la courbure devient très forte et la question se pose notamment de déterminer la tangente initiale.

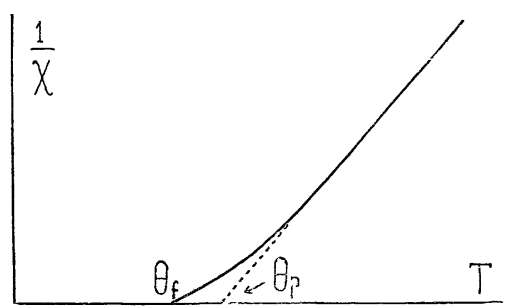

Fig. 1.

Des mesures préliminaires sur le fer m'ont montré que cette courbe était formée de portions de droites juxtaposées. Mais les expériences étaient difficiles et j'ai préféré les reprendre sur le nickel, avec une précision de beaucoup accrue: le point de Curie du nickel, $338^{\circ}$, est bien plus accessible que celui du fer : $770^{\circ}$.

Je me suis ainsi proposé d'étudier les propriétés magnétiques du nickel dans un intervalle de quelques degrés au-dessus du point de Curie, par la méthode classique d'attraction dans un champ non uniforme, au moyen de la balance de translation ( $\left.{ }^{1}\right)$. Deux points requièrent une attention spéciale $: 1^{\circ}$, la production et la mesure des températures; $2^{\circ}$, la production d'un champ magnétique non uniforme d'intensité convenable.

(1) Foex et Forrer, J. Phys., 1926, 7, p. 180.
2. Production et mesure des températures. Dans l'intervalle à étudier, la susceptibilité varie de 50 pour 100 , quand la température varie de $1^{\circ}$. Pour tirer pleinement parti des mesures de susceptibilité dont la précision relative atteint 1 pour 1000 , il faudrait définir et repérer les températures à $0^{\circ} 002$ près. A la température de $400^{\circ}$, il est possible de maintenir constante, à $0^{\circ} 03$ près, la température d'un four faiblement calorifugé et refroidi extérieurement par une circulation d'eau, tout au moins pendant une dizaine de minutes. Si la substance étudiée est bien isolée au milieu du four, les oscillations de température y sont fortement amorties. Le couple thermoélectrique étant placé dans la substance mème, à chaque instant, sa température ne doit différer que fort peu de la température moyenne de la substance. En fait, l'expérience montre que cette différence est inférieure à $0^{\circ}, 005$. J'ai employé un couple thermo-électrique d'Imphy (B.T.E-C.T.E), pratiquement inaltérable vers $400^{\circ}$ et fournissant une force électromotrice de $50 \mu \mathrm{V}$ par degré.

Pour la mesure des forces électromotrices j'ai construit un potentiomètre de grande précision déjà décrit ('). C'est un potentiomètre à déviation, à deux circuits indépendants qui permettent un auto-étalonnage très facile. Il permet de mesurer à $0,1 \mu \mathrm{V}$ près une force électromotrice de 20 millivolts. Les éléments étalons Weston de contrôle ont été placés dans un thermostat dont la température à été maintenue constante à $\pm 0^{\circ}, 1$ près.

Le couple a été étalonné, au moyen d'un certain nombre de points fixes, avec une précision estimée à $0^{\circ}, 2$. Sa caractéristique : $e=f\left(T^{\top}\right)$ est rectiligne à $0^{\circ}, 002$ près dans l'intervalle de température de $10^{\circ}$ où portent les mesures. La pente correspondante $\frac{\mathrm{d} e}{\mathrm{~d} T}$ est connue à 1,200 près. Au cours de ce travail, les températures seront données à $0^{\circ}, 00 \mathrm{I}$ près : ceci signifie seulement qu'un intervalle de température supérieur à $1^{\circ}$ est connu à $0, \breve{b}$ pour 100 près. Les valeurs absolues

(1) Néet, Bull. Soc. franç. Phys., 1933, 334, p. 20. 
des températures peuvent comporter une erreur de $\pm 0^{\circ}, 3$.

3. Production du champ magnétique non uniforme. - Dans la région étudiée, la susceptibilité est une fonction du champ magnétique dont on se propose de trouver la valeur limite dans un champ zéro. Il faut ainsi opérer dans une série de champs magnétiques faibles exactement connus, ce qui n'est possible qu'avec une bobine sans fer.

J'ai calculé approximativement une forme de bobine telle que l'attraction : $K \boldsymbol{H} \frac{\partial \boldsymbol{H}}{\partial x}$ présente un maximum très aplati suivant l'axe, en dehors de la bobine, pour permettre de loger le four commodément. J'ai construit une maquette reproduisant, au $\frac{1}{4}$ des dimensions linéaires, la bobine définitive et par retouches successives de cette maquette j'ai obtenu la section droite cicontre (fig. 2). L'enroulement est formé de deux sections comprenant chacune 9 ă mètres de tube de cuivre de $8 \mathrm{~mm}$ de diamètre. Les deux sections sont disposées en parallèle pour la circulation d'eau et en série pour le courant électrique. En régime permanent, on peut utiliser une intensité de 150 ampères.

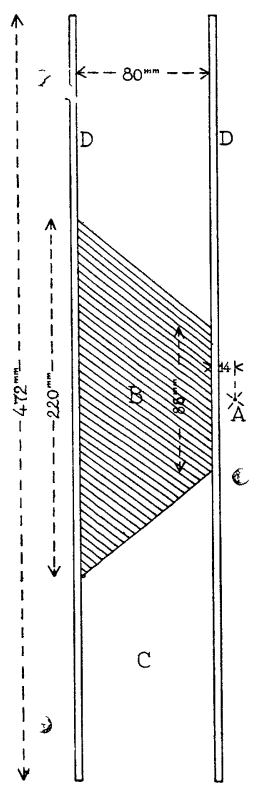

Fig. 2.

B, noyau en chêne; D, D, joues en laiton; C, espace occupé par l'enroulement; $A$, emplacement du maximum d'altraction.

Suivant l'axe, le maximum de $\boldsymbol{H} \frac{\partial \boldsymbol{H}}{\partial \boldsymbol{x}}$ est à $10 \mathrm{~mm}$ de la surface frontale de la bobine. De plus, la joue correspondante de la bobine, de $4 \mathrm{~mm}$ d'épaisseur, a été en taillée d'une ouver ture rectangulaire de $2 \times 1$ oั cm; on peut ainsi loger autour de la substance un four de $28 \mathrm{~mm}$ de diamètre. L'exploration du champ d'attraction a été faite au moyen d'une petite sphère de nickel de $2 \mathrm{~mm}$ de diamètre. Suivant l'axe, les résultats sont représentés par la figure 3 . Perpendiculairement à l'axe, les variations relatives de l'attraction sont inférieures à 1 pour 1000 , jusqu'à $4,5 \mathrm{~mm}$ de l'axe.

L'intensité du courant électrique dans la bobine est mesurée au potentiomètre avec une précision relative toujours supérieure à 0,5 pour 1000 .

Pour une valeur donnée du courant, le champ magnétique, à l'emplacement du maximum d'attraction. a été comparé au moyen d'un fluxmètre au champ d'un aimant étalon, lui-même mesuré à la balance de Cotton. On trouve ainsi : 7,02 gauss par ampère, à 1 pour 100 près.

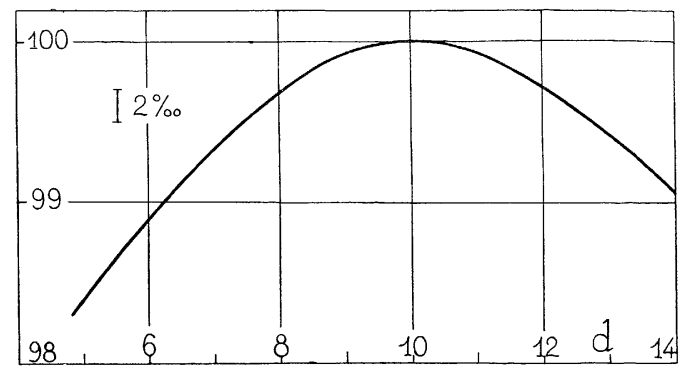

Fig. 3 - En ordonnée. les attractions en unités arbitraires; en abscisse, les distances en mm au plan de la bobine.

4. Etalonnage magnétique de l'appareil. - Dans l'étude du nickel, l'emploi de champs magnétiques faibles ne donne lieu à aucune difficulté, car les susceptibilités sont très grandes, mais l'étalonnage magnétique de la balance est délicat, car la susceptibilité du corps étalon, pyrophosphate de manganèse, est très petite vis-à-vis de celle du nickel. Il faut utiliser de grosses ampoules dont les parties périphériques sont nettement en dehors de la région du maximum d'attraction. Connaissant la topographie du champ d'attraction et la forme géométrique de l'ampoule supposée homogène, j'ai calculé qu'il fallait ajouter 3 pour 1000 à l'attraction mesurée. Deux ampoules de pyrophosphate, dont l'une avait été comparée à l'eau par M. Lallemand, ont fourni des résultats concordants. Les valeur's absolues des susceptibilités sont ainsi déterminées à 6 pour 1000 près et les valeurs relatives à 2 pour 1000 .

๖. Substances étudiées. - J'ai étudié deux échantillons de nickel, l'un préparé par la Mond Nickel Co, l'autre par W. C. Heraeus ('). Ultérieurement, ils seront respectivement désignés par les lettres $M$ et $H$. L'analyse donne les résultats suivants en pour 100 :

\begin{tabular}{|c|c|c|c|c|c|c|}
\hline & $\mathrm{Fe}$ & $\mathrm{Cu}$ & Co & $\mathrm{Mn}$ & DIVERS & $\mathrm{Ni}$ \\
\hline Nickel M ... & 0,18 & 0,01 & & & 11,05 & 99,76 \\
\hline Nickel H & 0,01 & 0,03 & $0,00 \mathrm{~s}$ & (1),005 & 0,04 & 99,91 \\
\hline
\end{tabular}

(') Voir Weiss et Forrer, Ann. de Phys., 1929, 12, p. 324. 
soit 2,4 pour 1000 d'impuretés dans le nickel M et 0,4 pour 1000 dans le nickel $\mathrm{H}$.

Les deux échantillons ont été pris sous la forme d'une sphère deti $\mathrm{mm}$ de diamètre, percée suivant un rayon d'un trou de $4,3 \mathrm{~mm}$ de profondeur et de $0,8 \mathrm{~mm}$ de diamètre qui servait de logement au couple thermoélectrique.

Avant les mesures, les deux sphères ont été recuites pendant $36 \mathrm{~h}$. à $800^{\circ}$ et refroidies en $24 \mathrm{~h}$. jusqu'à la température ordinaire.

6. Ordre des mesures. - J'ai constaté qu'il suffisait de quelques séries de variations thermiques pour stabiliser les échantillons, c’est-à-dire qu au cours d'une séance de mesures de $\mathbf{h}$., pendant laquelle la substance était toujours maintenue à une température supérieure au point de Curie, les mesures étaient réversibles et les substances n'évoluaient pas. Mais après avoir laissé la substance refroidir et séjourner longtemps à la température ordinaire ou à ùne température plus élevée mais toujours inférieure au point de Curie, on constatait quelquefois, un déplacement du point de Curie très faible d'ailleurs, n'atteignant qu'exceptionnellement $0^{\circ}, 1$.

La possibilité d'un tel déplacement, dans l'intervalle de deux séries de mesures, conduit au mode opératoire suivant :

$1^{\circ}$ Pour une série de températures fixes, on étudie les variations de la susceptibilité avec le champ magnétique, en ayant soin de faire toutes les mesures relatives à une mème température au cours d'une mème séance.

$2^{\circ}$ Pour un champ magnétique déterminé. on étudie les variations de la susceptibilité avec la température, en peu de séances aussi rapprochées que possible, et on utilise les coefificients de variation de la susceptibilité avec le champ magnétique, précédemment déterminés, pour ramener, s'il y a lieu, les observations au champ zéro.

7. Détermination des coefficients du champ démagnétisant. - La susceptibilité vraie \% est reliée à la susceptibilité apparente $\%_{\text {app. }}$ immédiatement donnée par l'expérience, par la formule $\left(^{1}\right)$.

$$
\frac{1}{\chi}=\frac{1}{\% \text { app. }}-n \text {, }
$$

où $n$ est le coefficient du champ démagnétisant. La mesure de la susceptibilité apparente, dans des conditions où la susceptibilité vraie est pratiquement infinie, au-dessous du point de Curie par exemple, permet de calculer $n$.

Le tableau I, relatif au nickel $\boldsymbol{H}$, montre que $\boldsymbol{n}$ ainsi déterminé est effectivement indépendant de la température et du champ magnétique.

J'ai adopté les valeurs suivantes:

Nickel M : $\quad n=34,70, \quad$ Nickel H : $\quad n=3$ อ̆,58.

(1) Les valeurs de $\%, \%$ app. etc., sont toujours exprimées en C. G. S. et rapportées à l'unité de masse.
Tablead I.

\begin{tabular}{|c|c|c|}
\hline$T$ & $H$ & $\frac{1}{\chi_{\text {app. }}}=n$ \\
\hline $332^{\circ} 24$ & $\begin{array}{l}31,7 \\
60,1\end{array}$ & $\begin{array}{l}35,53 \\
35,64\end{array}$ \\
\hline $33^{\circ} 3 \ddot{3}$ & $\begin{array}{r}59,2 \\
115,0 \\
201,6\end{array}$ & $\begin{array}{l}35,56 \\
35,4 \times \\
35,56\end{array}$ \\
\hline $3 \check{3} 3^{\circ} 68$ & $\begin{array}{l}31,6 \\
59,8\end{array}$ & $\begin{array}{l}3 \check{3}, 57 \\
3 \check{,}, 40\end{array}$ \\
\hline $354^{\circ} 36$ & $\begin{array}{r}60,2 \\
117,0\end{array}$ & $\begin{array}{l}35,75 \\
35, \text { כ0 }\end{array}$ \\
\hline $355^{\circ} 7$ 亿 & $\begin{array}{l}31,3 \\
58,9\end{array}$ & $\begin{array}{l}3 \check{5}, 577 \\
3 \check{5}, 53\end{array}$ \\
\hline
\end{tabular}

Le champ magnétique interne réellement agissant $h$, est relié au champ extérieur $\boldsymbol{H}$ par la formule:

$$
h=H \frac{\chi_{\text {app. }}}{\%} \text {. }
$$

Dans la suite, je ne donnerai que les valeurs de la susceptibilité vraie et du champ réellement agissant, sauf indication contraire.

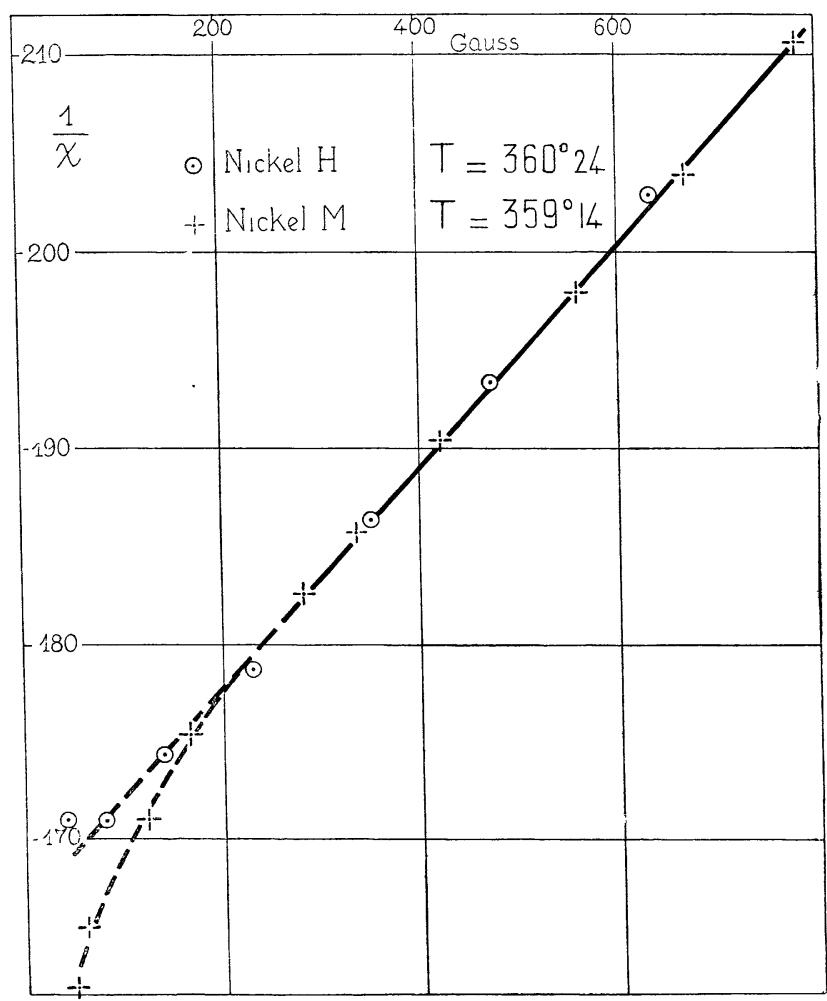

Fig. 4 . 
La susceptibilité dans un intervalle de $2^{\circ} 5$ au-dessus du point de Curie $\theta_{t}$ - Le tableau II et les figures 1 et 4 bis rassemblent quelques valeurs de $\chi$ en fonction de $h$.

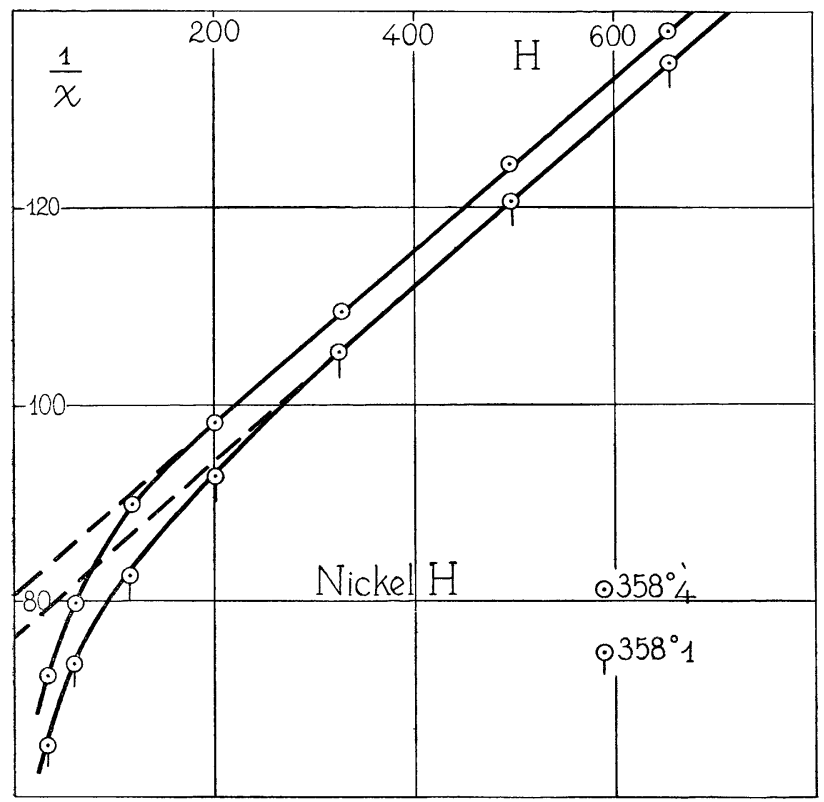

Fig. 4 bis.

Le théorie ( $\left.{ }^{1}\right)$ indique que pour des champs magnétiques suffisamment faibles, $\frac{1}{\gamma}$ est de la forme :

$$
\frac{1}{\chi}=\frac{1}{\chi_{0}}+c h^{2} .
$$

Une telle variation est incompatible avec les résultats expérimentaux, puisque, même avec une représentation en $h$, les courbes sont concaves vers l'axe des champs. Il est ainsi impossible de définir une susceptibilité initiale $\%$.

Sauf pour des champs très faibles, la courbe du nickel $M$, à $359^{\circ}, 140$, est confondue avec la courbe du nickel $\mathrm{H}$, à $360^{\circ}, 240$, ce qui indique que la différence entre les points de Curie de ces deux substances est très approximativement $1^{\circ} 10$.

9. Variations de la susceptibilité avec le champ magnétique pour des températures supérieures de plus de $2^{\circ} 5$ au point de Curie (lableaux III et IV, fig. 5). - Le problème s'éclaircit. J'ai tracé cette fois-ci $\frac{1}{\chi}$ en fonction de $h^{2}$. On voit pour le nickel $H$, que dans des champs supérieurs à 300 gauss, une relation de la forme(1) est vérifiée; pour le nickel $M$, elle n'est vérifiée que dans des champs supérieurs à

(') Néel, J. Phys., 1934, s. 8, t. 5, p. 110.
Tableau II

\begin{tabular}{|c|c|c|}
\hline$T$ & $h$ & $\frac{1}{x}$ \\
\hline $3 . ั 90140$ & $\begin{array}{r}\text { Vickel } M \\
49,6 \\
63,3 \\
123, \breve{5} \\
169,4 \\
281, \check{5} \\
335,9 \\
423,1 \\
508,6 \\
669,8 \\
781,9\end{array}$ & $\begin{array}{l}162,2 \\
165,1 \\
171,0 \\
175,4 \\
182,6 \\
185,7 \\
190,4 \\
197,9 \\
203,9 \\
910,6\end{array}$ \\
\hline $358^{\circ} 100$ & $\begin{array}{r}\text { Yickel } H \\
14,3 \\
30,3 \\
65,5 \\
123,0 \\
211,1 \\
350,2 \\
482,7\end{array}$ & $\begin{array}{l}29,78 \\
38,05 \\
47,10 \\
57,17 \\
70,35 \\
85,02 \\
99,06\end{array}$ \\
\hline $358^{\circ} 400$ & $\begin{array}{r}16,1 \\
33,3 \\
69,3 \\
127,7 \\
220,7 \\
353,8 \\
487,3\end{array}$ & $\begin{array}{r}36.92 \\
44,32 \\
53,19 \\
62, \text {.ั. } \\
73,95 \\
88,92 \\
102,35\end{array}$ \\
\hline $360^{\circ 240}$ & $\begin{array}{r}41,3 \\
80,0 \\
139,9 \\
228,4 \\
351,7 \\
472,7 \\
635.3\end{array}$ & $\begin{array}{l}171.0 \\
1710 \\
174,4 \\
178,7 \\
186,4 \\
193,4 \\
202,9\end{array}$ \\
\hline
\end{tabular}

600 gauss. Pour les champs inférieurs à ces limites, la susceptibilité prend des valeurs anormalement grandes, d'autant plus grandes que le champ est plus faible. Toutes choses égales d'ailleurs, l'effet est plus marqué sur le nickel M.

Ce phénomène est nouveau.

Le phénomène normal, régulier est certainement la variation donnée par la formule (1), d'une part, à cause des considérations théoriques qui l'appuient et d'dutre part, parce que les coefficients $c$, considérés comme des fonctions de $\chi_{0}$ sont les mêmes pour les deux échantillons de nickel, tandis que la courbure initiale, différente dans les deux cas, prend l'aspect d'un phénomène parasite. 


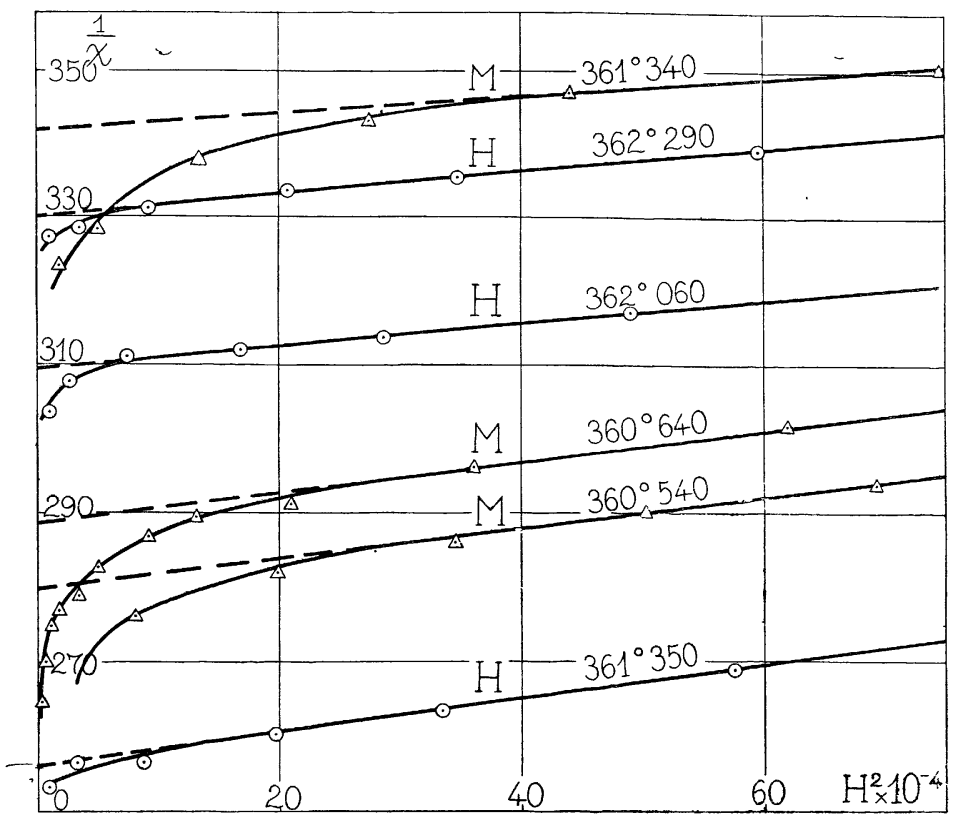

Fig. 5.

Tableau III. - Nickel $M$.

\begin{tabular}{|c|c|c|c|}
\hline$T$ & $h$ & $\frac{1}{x}$ & $h^{2} \times 10^{-3}$ \\
\hline $360^{\circ}$ อั40 & $\begin{array}{l}293,1 \\
444,6 \\
\div 88,9 \\
\div(18,11 \\
832,4\end{array}$ & $\begin{array}{l}276,4 \\
282,1 \\
28 \%, 9 \\
-911,3 \\
\div 94,1\end{array}$ & $\begin{array}{r}86 \\
198 \\
346 \\
\dddot{301} \\
693\end{array}$ \\
\hline $360^{\circ} 640$ & $\begin{array}{r}53,8 \\
68,1 \\
103,7 \\
132,0 \\
181,4 \\
221,3 \\
303,3 \\
361,7 \\
457,3 \\
599,7 \\
786,8\end{array}$ & $\begin{array}{l}264,4 \\
270,3 \\
27 \%, 0 \\
277,1 \\
274,0 \\
282,9 \\
481,8 \\
289,7 \\
291,2 \\
496,2 \\
301, x\end{array}$ & $\begin{array}{r}3 \\
5 \\
11 \\
17 \\
33 \\
30 \\
92 \\
131 \\
209 \\
360 \\
619\end{array}$ \\
\hline $361^{\circ} 340$ & $\begin{array}{l}132,9 \\
223,9 \\
363,3 \\
522,6 \\
6+33,3 \\
866,4\end{array}$ & $\begin{array}{l}323,6 \\
328,4 \\
338,0 \\
343,2 \\
347,1 \\
350,4\end{array}$ & $\begin{array}{r}18 \\
50 \\
132 \\
473 \\
440 \\
731\end{array}$ \\
\hline
\end{tabular}

Il faut sans doute supposer que, malgré des recuits prolongés, les substances ne sont pas homogènes. ¿uelques millièmes de la masse de l'échantillon, avec
Tablbau IV. - Nickel $\boldsymbol{H}$.

\begin{tabular}{|c|c|c|c|}
\hline$T$ & h & $\frac{1}{\%}$ & $h^{2} \times 10^{-3}$ \\
\hline $3+i 1^{\circ} 3 \check{0} 0$ & $\begin{array}{l}102,8 \\
180,0 \\
294,0 \\
443,3 \\
578,2 \\
758,5\end{array}$ & $\begin{array}{l}253,3 \\
256,5 \\
256,7 \\
260,3 \\
263,5 \\
269,2\end{array}$ & $\begin{array}{r}11 \\
32 \\
86 \\
196 \\
334 \\
37 \%\end{array}$ \\
\hline $362^{\circ} 290$ & $\begin{array}{l}106,0 \\
184,3 \\
3113,0 \\
453,7 \\
590,0 \\
772,0\end{array}$ & $\begin{array}{l}327,4 \\
328,6 \\
331,3 \\
333,7 \\
33 a, 4 \\
339,2\end{array}$ & $\begin{array}{r}11 \\
34 \\
92 \\
206 \\
348 \\
\check{2} 96\end{array}$ \\
\hline $362^{\circ} 060$ & $\begin{array}{r}9: 3,1 \\
166,2 \\
271,7 \\
407,5 \\
535,3 \\
700,0\end{array}$ & $\begin{array}{l}303,8 \\
307,8 \\
311,2 \\
312,0 \\
313,7 \\
317,1\end{array}$ & $\begin{array}{r}9 \\
28 \\
74 \\
166 \\
287 \\
491\end{array}$ \\
\hline
\end{tabular}

un point de Curie de $5^{\circ}$ plus élevé que le reste, suffiraient à produire des effets analogues. J'attribue donc la courbure initiale à une sorte d'étalement du point de Gurie. L'origine en est sans doute complexe, néanmoins les impuretés doivent jouer un rôle, car c'est le nickel M le plus impur, qui présente les anomalies les plus grandes. 
Très près du point de Curie, c'est-à-dire à l'intérieur de l'étalement, les effets de mélange masquent complètement le phénomène idéal relatif à une substance pure. C'est pourquoi il est impossible, à ce point de vue, de tirer parti des résultats du paragraphe précédent.

Tableau $\mathrm{V}$.

\begin{tabular}{|c|c|c|c|c|}
\hline NICKEL & $T$ & $c \times 10^{6}$ & $\frac{1}{\gamma_{0}}$ & $\sigma_{0}$ \\
\hline M & $\begin{array}{r}360^{\circ} 640 \\
540 \\
361^{\circ} 340 \\
359^{\circ} 733 \\
940 \\
360^{\circ} 314 \\
362^{\circ} 350 \\
520 \\
900\end{array}$ & $\begin{array}{l}21,4 \\
20,7 \\
11,8 \\
3 \breve{3}, 8 \\
2 \ddot{3}, 4 \\
26,4 \\
7,0 \\
8,8 \\
\check{3}, 4\end{array}$ & $\begin{array}{l}288,4 \\
279,8 \\
341,8 \\
222,0 \\
238,8 \\
262,0 \\
422,2 \\
431,8 \\
466,8\end{array}$ & $\begin{array}{l}68,6 \\
71,7 \\
78,6 \\
6 \checkmark, 0 \text { ? } \\
75,3 ? \\
67,6 \text { ? } \\
\times 3,8 \text { ? } \\
73,3 \text { ? } \\
86,9 \text { ? }\end{array}$ \\
\hline H & $\begin{array}{r}362^{\circ} 060 \\
290 \\
361^{\circ} 350\end{array}$ & $\begin{array}{l}1 \supset, 4 \\
14,5 \\
23,4\end{array}$ & $\begin{array}{l}309,8 \\
330,5 \\
205,8\end{array}$ & $\begin{array}{l}75,5 \\
73,1 \\
73,5\end{array}$ \\
\hline
\end{tabular}

Le tableau $\mathrm{V}$ donne quelques déterminations de $c$, et de $\frac{1}{\chi_{0}}$, dans l'intervalle de température où ces déterminations sont possibles. Cet intervalie est d'ailleurs restreint, dans la limite des champs magnétiques employés, du côté des basses températures pour la raison énoncée ci-dessus et du còté des hautes températures, parce que $c$ variant en raison inverse du carré de la distance au point de Curie ( ${ }^{1}$ ), les variations relatives de susceptibilité, pour une même variation de champ, varient en raison inverse du cube de la distance au point de Curie et décroissent ainsi excessivement vite.

10. Interprétation du coefficient c. - $\mathrm{Si}$ l'on attribue le paramagnétisme du nickel à des porteurs de moment angulaire $\mathrm{S}=\frac{1}{2}$, uniquement dû au spin, j'ai montré $\left({ }^{2}\right)$ que $c$ est relié à $\sigma_{0}$, moment total obtenu en alignant les porteurs parallèlement, par la formule:

$$
c=\frac{\lambda}{\sigma_{0}^{2}} \frac{\%_{0}+n}{\%_{0}^{2}}
$$

$n$ et $\lambda$ se déterminent par l'étude des variations de la susceptibilité initiale avec la température.

On trouve $n=2400$ et $\lambda=3,11$ entre $360^{\circ}$ et $370^{\circ}$.

La formule 2 permet de calculer $\sigma_{0}$ (dernière colonne du tableau 5 ). Pour le nickel $M$, par suite de l'importance du terme perturbateur signalé dans le paragraphe

(1) NéEl. J. Phys., 1934, s. 7, t. 5, p. 110.

(2) Néel, J. de Phys., 1934, s. 7, t. 5, p. 106. précédent, les valeurs de $\sigma_{0}$ sont moins bien groupées que pour le nickel H. Néanmoins, la valeur moyenne de $\sigma_{0}$ relative au nickel $M: 74,8$ est très voisine de la valeur moyenne 74,0 fournie par l'étude du nickel $H$. Le bon accord de ces deux déterminations justifie le mode de discussion adopté. L"aimantation maximum moyenne utilisée dans ces déterminations de $c$ était $\sigma=2,5$.

Ces résultats joints à ceux que fournit l'étude du nickel dans les champs forts, d'après les expériences de Weiss et Forrer $\left({ }^{1}\right)$, montrent que $c_{0}$ est, en réalité, une fonction lentement variable de l'aimantation $\sigma$, qui tend vers $\sigma_{0}=7 \bar{i}$, pour $\sigma=0$. Cette valeur conduit à altribuer au porteur élémentaire le moment $\mu=5782$, très voisin du magnéton de Bohr: $u_{B}=5564$. L'hypothèse initiale énoncée au début de ce paragraphe est ainsi confirmée.

11. La susceptibilité initiale en fonction de la température. - Cette étude ayant déjà été faite avec précision dans un large intervalle de température, je ne la reprendrai pas. Je me propose simplement, dans un intervalle de température voisin du point de Curie, de déterminer l'allure de la variation.

Je ne donne ainsi que quelques résultats expérimentaux obtenus entre $361^{\circ} 6$ et $370^{\circ}$ pour le nickel $H$, dans un champ magnétique extérieur de 470 gauss. Les valeurs $\frac{1}{\gamma_{a}}$ n'ont pas été corrigées du champ démagnetisant. Ces valeurs ont été ramenées au champ 0 , en utilisant les valeurs de $c$ données par la formule (2), avec $\sigma_{0}=7-0$. Aux températures les plus basses la correction est incertaine car le graphique $\frac{1}{\chi}=f\left(h^{2}\right)$ ne présente plus qu'une partie rectiligne évanouissante. Les valeurs $\frac{1}{\chi_{0}}$, ainsi déterminées, corrigées du champ démagnétisant, sont données dans le tableau VI. Elles correspondent à cinq séries distinctes de mesures.

Reportées sur un graphique, ces valeurs se placent sur une courbe régulière. Pour préciser ce point, j'ai classé les points expérimentaux, par ordre de température croissante, en huit groupes, et j'ai calculé $T$ moyen et $\frac{1}{\chi_{0}}$ moyen pour chaque groupe $\left(^{2}\right)$ (tableau VII). Le point fictif, dont les coordonnées sont ainsi calculées, doit être très voisin d'un point réel puisqu'à l'intérieur d'un groupe la courbure est tout à fait négligeable.

Pour représenter graphiquement les résultats à grande échelle, j'ai porté en fonction de $T$, les différences :

$$
\Delta=\frac{1}{\chi_{0}}-\frac{1}{\chi_{1}} \text { avec } \frac{1}{\chi_{1}}=81\left(T-358^{\circ} 140\right) .
$$

(l) Ann. de Phys., 1926, 5, p. 153.

(2) Dans le tableau VII, j'ai désigné par $n$ le nombre des poinls de chaque groupe. 


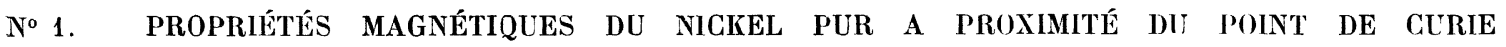

TablaAu VI. - Nickel $H-\frac{1}{\chi}$ mesuré dans $H=470 G$, puis ramené au champ $O$.

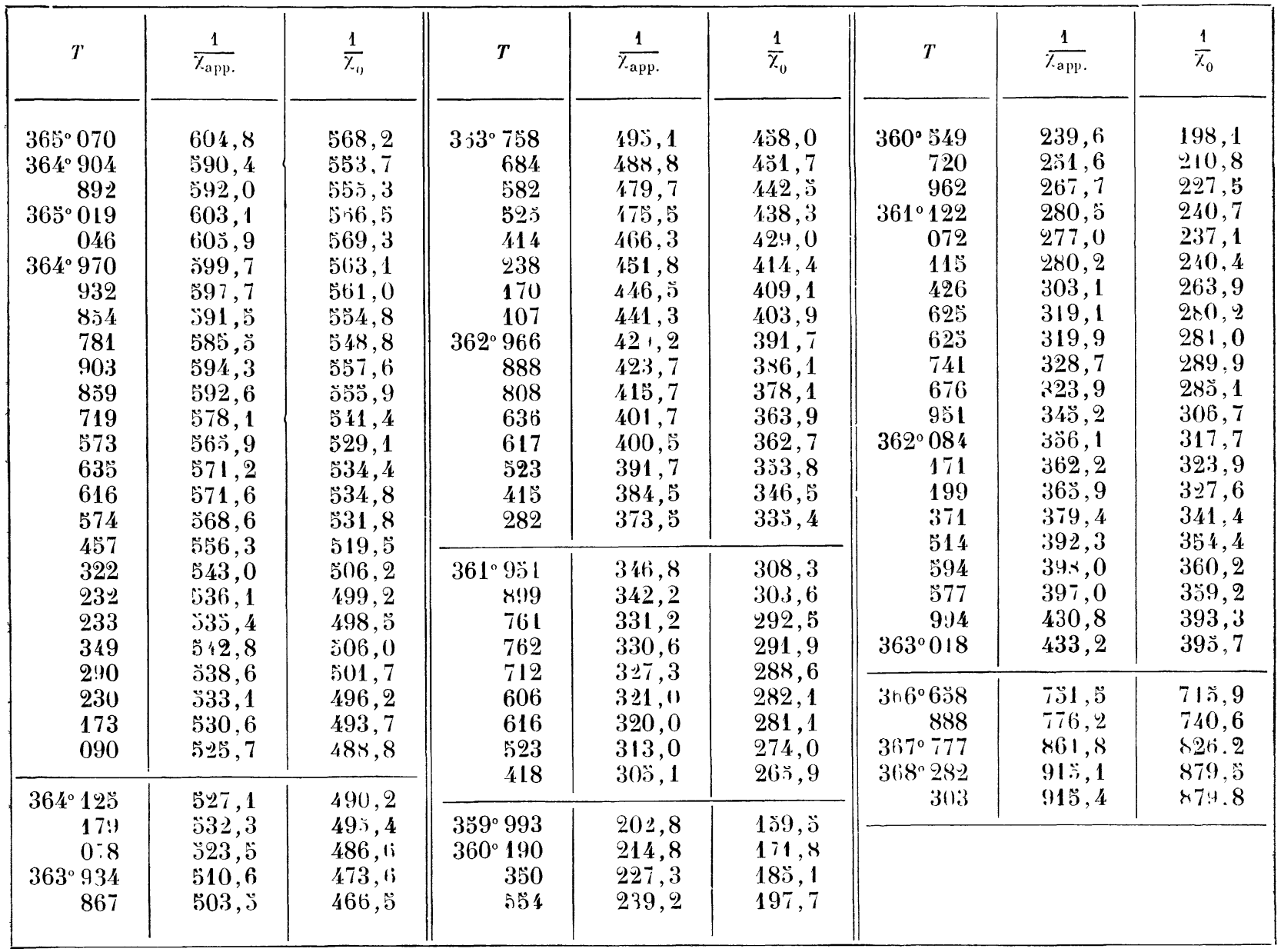

Tableau VII.

\begin{tabular}{|c|c|c|c|}
\hline$n$ & $T$ & $\frac{1}{\gamma_{01}}$ & $\Delta$ \\
\hline 6 & $360^{\circ} 393$ & 187,16 & $\begin{array}{r}4,63 \\
+\quad 4\end{array}$ \\
\hline 7 & $361^{\circ} 234$ & 249,97 & $-0,68$ \\
\hline 13 & 770 & 292,97 & $-\quad 110$ \\
\hline 11 & $362^{\circ} 145$ & 348.09 & $-\quad 0,6 \mathrm{a}$ \\
\hline 15 & $363^{\circ} 330$ & 422,12 & $\begin{array}{r}1,69 \\
+\quad 1,\end{array}$ \\
\hline 13 & $364^{\circ} 256$ & 501,06 & $\begin{array}{r}5,62 \\
+\quad 5\end{array}$ \\
\hline 15 & 851 & 552,93 & $\begin{array}{r}+ \\
+\quad 9,30\end{array}$ \\
\hline$\dddot{3}$ & $367^{\circ}$ อั81 & 808,42 & $+43,70$ \\
\hline
\end{tabular}

La figure 6 montre qu'on oblient ainsi une courbe parfaitement régulière, sans aucune trace de parties rectilignes. Ainsi, même dans les champs faibles, il n'y a pas de rectification; le " principe d'uriformisa-

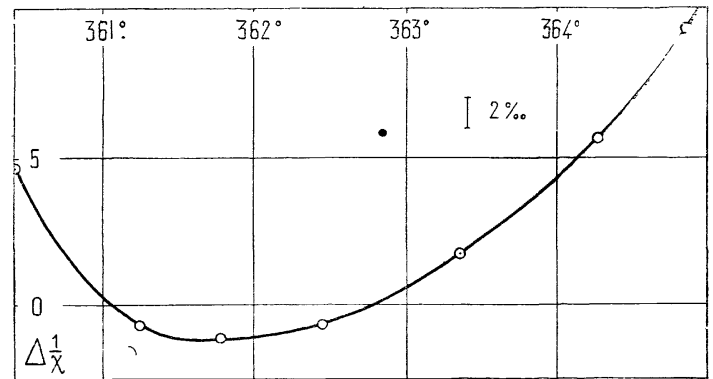

Fiğ. 6.

tion " (') ne joue pas. La courbure continue que l'on observe est bien celle que laisse prévoir l'influence des fluctuations du champ moléculaire (').

(1) P. Welss J. Physs, 1911, .je s, t. 1, p. 900 et Congrès Int. d'Electr. t. I, rapp. 15, 1932

G. För. J. Phys., 1931, Te s., t. 2, p. 353.

(2) NÉEL. Annales de I'hys, 1932, t. 17. p. วั. 
La formule parabolique suivante permet de représenter les résultats expérimentaux entre $366^{\circ} 4$ et $367^{\circ} 8$, dans un intervalle où la susceptibilité varie dans le rapport de 1 à 4 ;

$\frac{1}{\% 0}=295,4+81\left(T-361^{\circ} 8\right)+1,25\left(T-361^{\circ} 8\right)^{2}$.

L'écart entre les valeurs expérimentales et les valeurs caleulées d'après cette formule est de l'ordre de quelques millièmes sauf pour le point le plus bas. $\left(T=360^{\circ} 393\right)$ où l'écart atteint exceptionnellement 1,8 pour 100 .

D'après la formule (3) on calcule la température du point de Curie $\Theta=357^{\circ} 92$ et la tangente au point de Curie :

$$
\left(\frac{\partial T}{\partial \frac{1}{\%}}\right)=71,3
$$

Ces valeurs ne sont pas excessivement sûres car l'extrapolation porte sur un intervalle de plus de deux degrés, dans lequel il se pourrait que l'allure de la courbe changeât.

12. Remarque sur la détermination des points de Curie. - La méthode de détermination du point de Curie, exposée plus haut, conduit, dans le cas du nickel H par exemple, à n'utiliser que les observations dans des champs supérieurs à 300 gauss. Elle fournit correctement un point de Curie moyen qui est le centre de gravité des points de Curie des différents éléments qui constituent la substance. Au 'contraire, lorsqu'on opère seulement dans un champ magnétique faible,
100 gauss par exemple, l'élude des courbes fournit un point de Curie plus élevé que le point de Curie moyen et s'il était possible d'expérimenter dans un champ infiniment faible on obtiendrait ainsi le point de Curie des portions de substance qui ont le point de Curic le plus élevé.

Bref, une détermination correcte et précise du point de Curie nécessite la connaissance complète de l'équation d'état magnétique au voisinage de celui-ci. C'est à celte condition qu'il est possible de le définir à $0^{\circ} 1$ près.

14. Conclusions. - $1^{\circ}$ L'étude du nickel dans les champs faibles, au voisinage $\mathrm{du}$ point de Curie, montre que $\frac{1}{\gamma}$ varie d'une manière régulière avec la température, ainsi que sa dérivée première. Le principe d'uniformisation ne joue pas : il n'y a pas d'états magnétiques différents.

$2^{\circ}$ L'étude des propriétés magnétiques très près du point de Curie, montre l'existence probable d'un étalement du point de Curie, de l'ordre du degré, d'autant plus grand que le nickel est moins pur.

$3^{\circ}$ Les valeurs de $c=\frac{\partial \frac{1}{\chi}}{\partial H^{2}}$, en fonction de $T$ se raccordent aux déterminations faites d'après les expériences de Weiss et Forrer et confirment l'attribution, au porteur élémentaire de magnétisme de nickel, d'un moment égal au magnéton de Bohr.

$4^{\circ}$ La tangente à la courbe $\frac{1}{\gamma}=f(T)$ possède, au point de Curie, la valeur 71,3. 